\title{
Lateral lumbar interbody fusion (LLIF): Technique and outcomes
}

\author{
Chester E Sutterlin III, ${ }^{1,2}$ MD, FACS; Jane E Luscombe ${ }^{3}$, RN; Jerry Day ${ }^{3}$, MD, FACS, FRACS; \\ Arvind Dubey ${ }^{4,5}, \mathrm{MD}$ \\ ${ }^{1}$ Department of Neurosurgery, University of Florida, Florida, USA \\ ${ }^{2}$ Spinal Health International, Longboat Key, Florida, USA, \\ 3/llawarra Neurosurgery, Wollongong, Australia \\ ${ }^{4}$ Neurosurgery Department, Royal Hobart Hospital, Hobart, Australia \\ ${ }^{5}$ Hobart Brain and Spine Centre, Hobart, Australia \\ Corresponding author \\ Chester E Sutterlin III, MD, FACS \\ Email: csutterlin3@hotmail.com \\ Received 19 Nov 2018 \\ Accepted 10 Dec 2018
}

\section{Introduction}

Historically, an interbody device (IBD) has consisted of morselized autograft ${ }^{1}$, structural autograft, structural allograft, stainless steel ball, threaded titanium cage, polyetheretherketone (PEEK) cage, and more recently 3D printed titanium cage with bioactive surface characteristics and bony ingrowth into IBD interstices. These IBD's have been inserted through a variety of approaches, both by open technique and by minimally invasive surgical (MIS) technique. Traditionally, the most common procedures have been posterior lumbar interbody fusion (PLIF), transfacetal lumbar interbody fusion (TLIF), and anterior lumbar interbody fusion (ALIF). Obviously, both PLIF and TLIF are posterior approaches, and ALIF is an anterior approach. More recent approaches in the retroperitoneal space anteriorly are oblique lumbar interbody fusion (OLIF) anterior to the psoas muscle, and lateral lumbar interbody fusion (LLIF) which is a transpsoas procedure. LLIF is the subject of this manuscript. The LLIF technique utilizing K2M's Ravine retractor system and K2M's lateral IBD's, Aleutian (PEEK) and Cascadia (3D printed titanium) will be described (K2M, Leesburg, VA USA). Bone graft substitute, iFactor (Cerapedics, Colorado USA), was used in all cases. No autograft was harvested from the iliac crest, but local morselized autograft was utilized if available. The clinical outcomes for LLIF using these implants and instruments will be reported.

\section{Methods}

The key points of the Ravine LLIF technique as outlined in the $\mathrm{K} 2 \mathrm{M}$ procedure manuals are summarized in this manuscript. The clinical outcomes from Dr. Dubey's practice are also summarized. The principal indications for surgery were adults with multilevel degenerative disc disease, with or without lumbar deformity and/or spinal imbalance, with or without neurologic deficit. Fractures, dislocations, tumors, and infections were excluded. Pediatric and adolescent deformity patients were excluded. Data on all consecutive patients was collected over a 4-year period from 2012-2016.

\section{Technique}

The patient is placed on a radiolucent operating table in the lateral decubitus position, usually with the left side up. The chest, pelvis, and legs are taped to secure the patient during surgery with the chest above and the pelvis below the pivot point of the table. The pivot point is angled to increase the distance between lower ribs and iliac crest (Fig. 1). Horizontal fluoroscope orientation is first used to assure proper AP view of the operative level of the lumbar spine (Fig. 2). Vertical fluoroscope orientation is used to assure proper lateral view (Fig. 3). The skin is then marked for orientation and incision, and the area is prepared and draped. The surgeon should visualize the underlying anatomy in his mind's eye (Fig.4). 


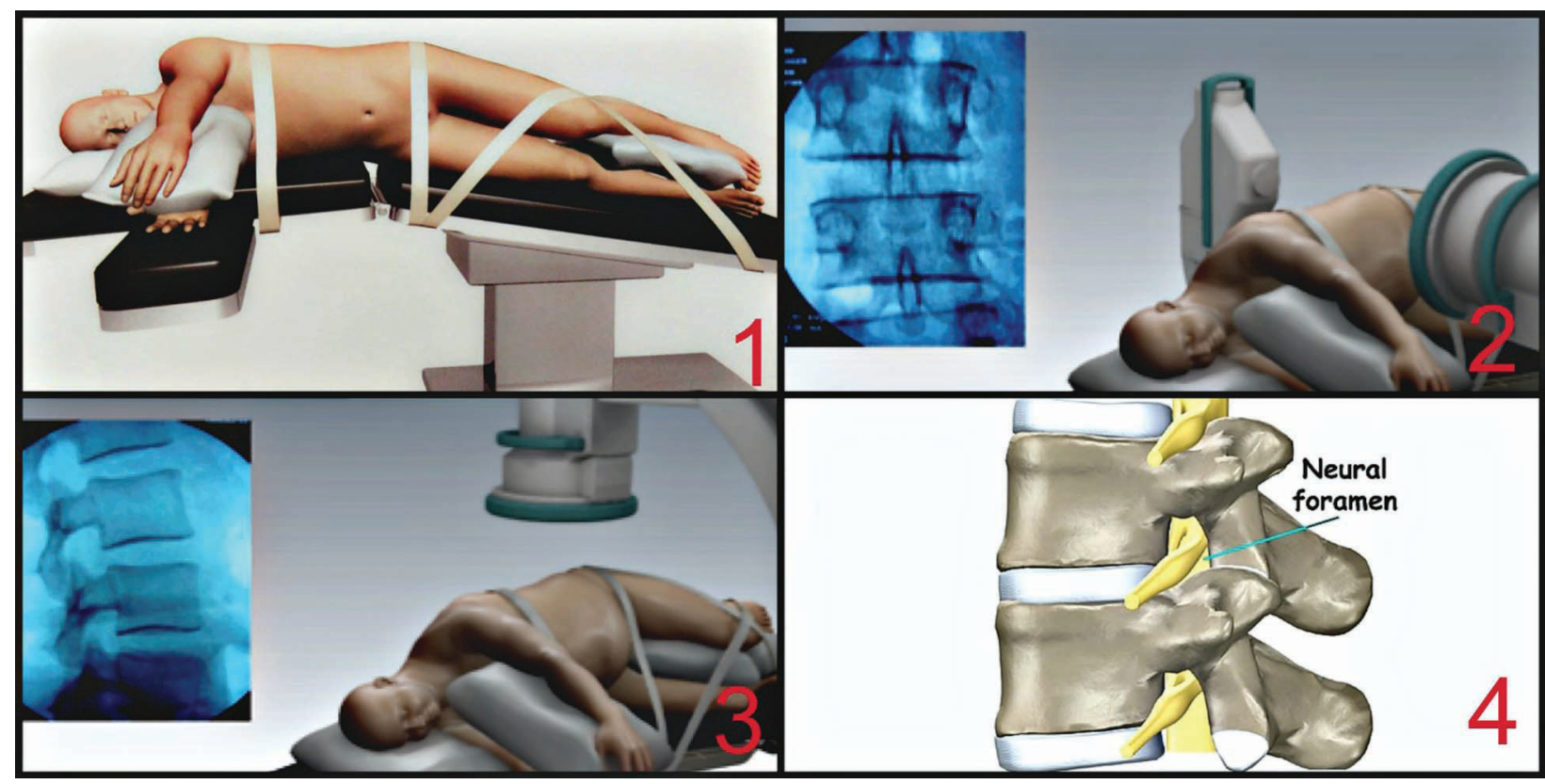

Once the skin incision is made, the dissection is carried through the muscle and fascial layers until the retroperitoneal space is reached. Blunt finger dissection is used to identify the psoas muscle (Fig.5). If the minimally-invasive surgical (MIS) approach is taken, then a series of MIS dilators are utilized to safely traverse the psoas muscle (Fig.6) using intraoperative neuromonitoring (IONM) to protect neural structures (Fig. 7). The Ravine MIS retractor system is then inserted and attached to the spinal vertebrae to give a secure attachment to the spine (Fig. 8, 9, 10).

Clear visualization of the disc space is achieved and greatly improved by fiberoptic light attachments. Check the final retractor location on vertical fluoroscopic (lat.) view prior to entering the disc space. The remainder of the procedure is performed with fluoroscopic guidance in the horizontal (AP) view with Ravine retractor firmly attached to the

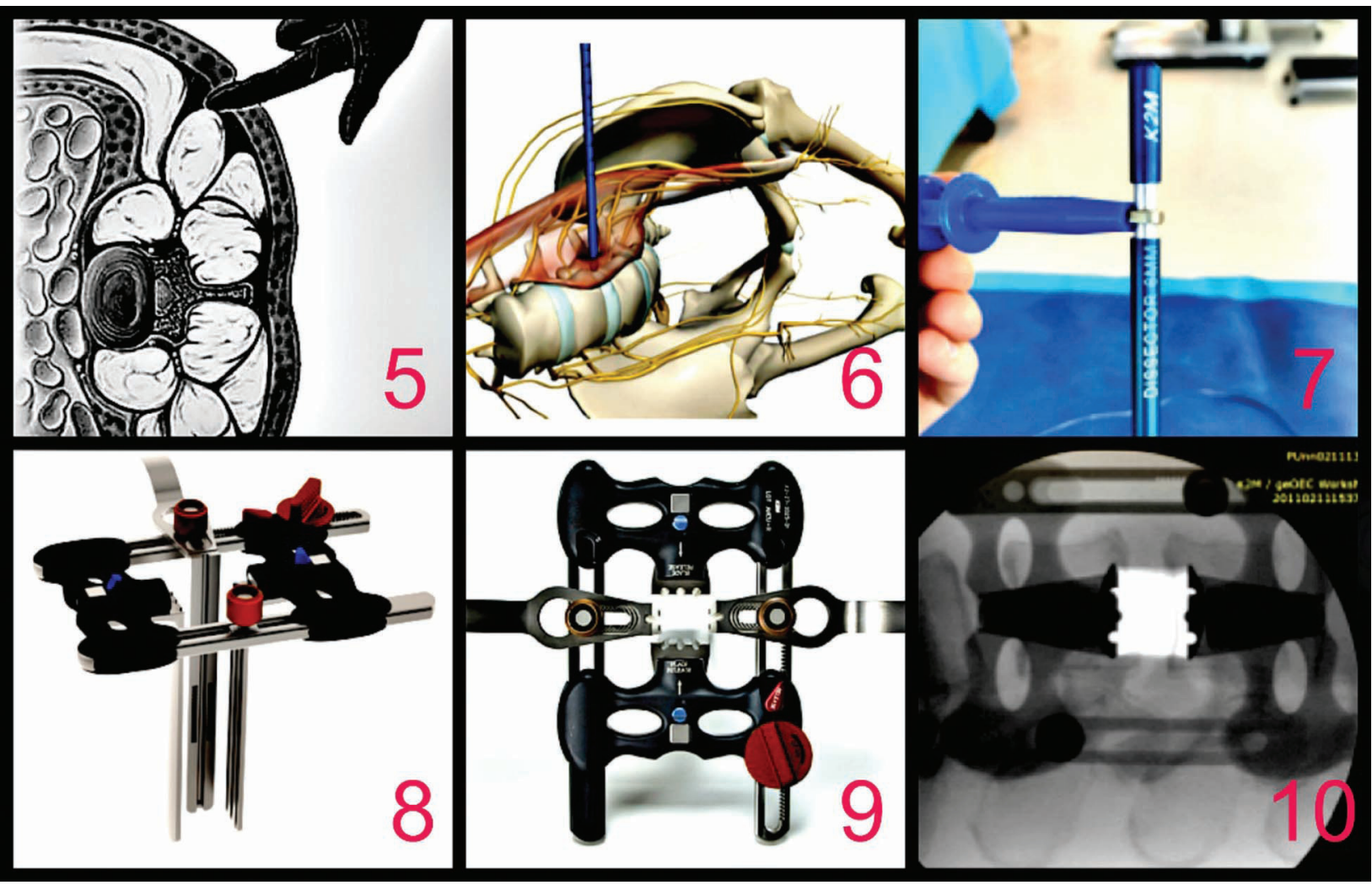


lumbar spine (Fig. 11). Supplemental attachment to the operative table is not necessary, although hardware is available for this purpose. The disc is then entered through an annular window, nucleus pulposus is removed, endplates are prepared by cartilage removal, and dilators and trials are utilized sequentially to determine proper implant size (Fig. 12). An LLIF interbody device (IBD) is inserted. K2M Aleutian polyetheretherketone (PEEK) implants can
IBD placement. The details of the technique can be accessed on the K2M website, <K2M.com>.

\section{Outcome results}

Asad, Dubey, Dubey, and Sutterlin reported on a consecutive series of 32 patients operated between 2012-2016 with average 13.2 months follow-up². The primary indication for surgery was degenerative deformity (scoliosis) with/without spinal imbalance

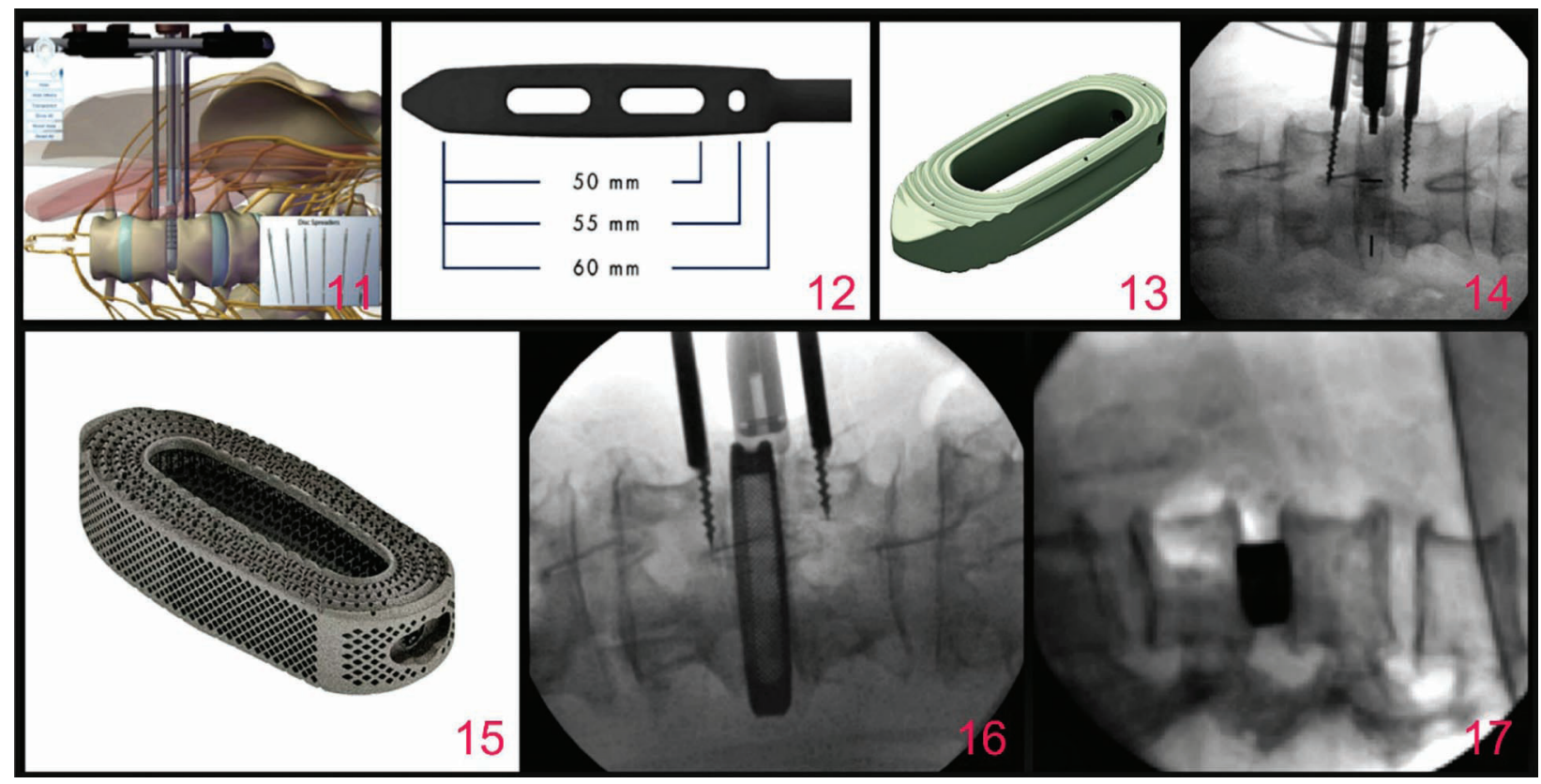

be inserted (Fig. 13 and 14). Alternatively, K2M 3D-Printed Titanium Cascadia implants can be used (Fig. 15, 16, and 17).

The Ravine retractor is removed and final fluoroscopic evaluation is made to assure proper and with/without neurologic deficit. A few patients in the series had 1-3 level degenerative disc disease without deformity or imbalance, and may or may not have had neurologic deficit. All patients received LLIF (K2M Ravine with Aleutian Lat (PEEK)

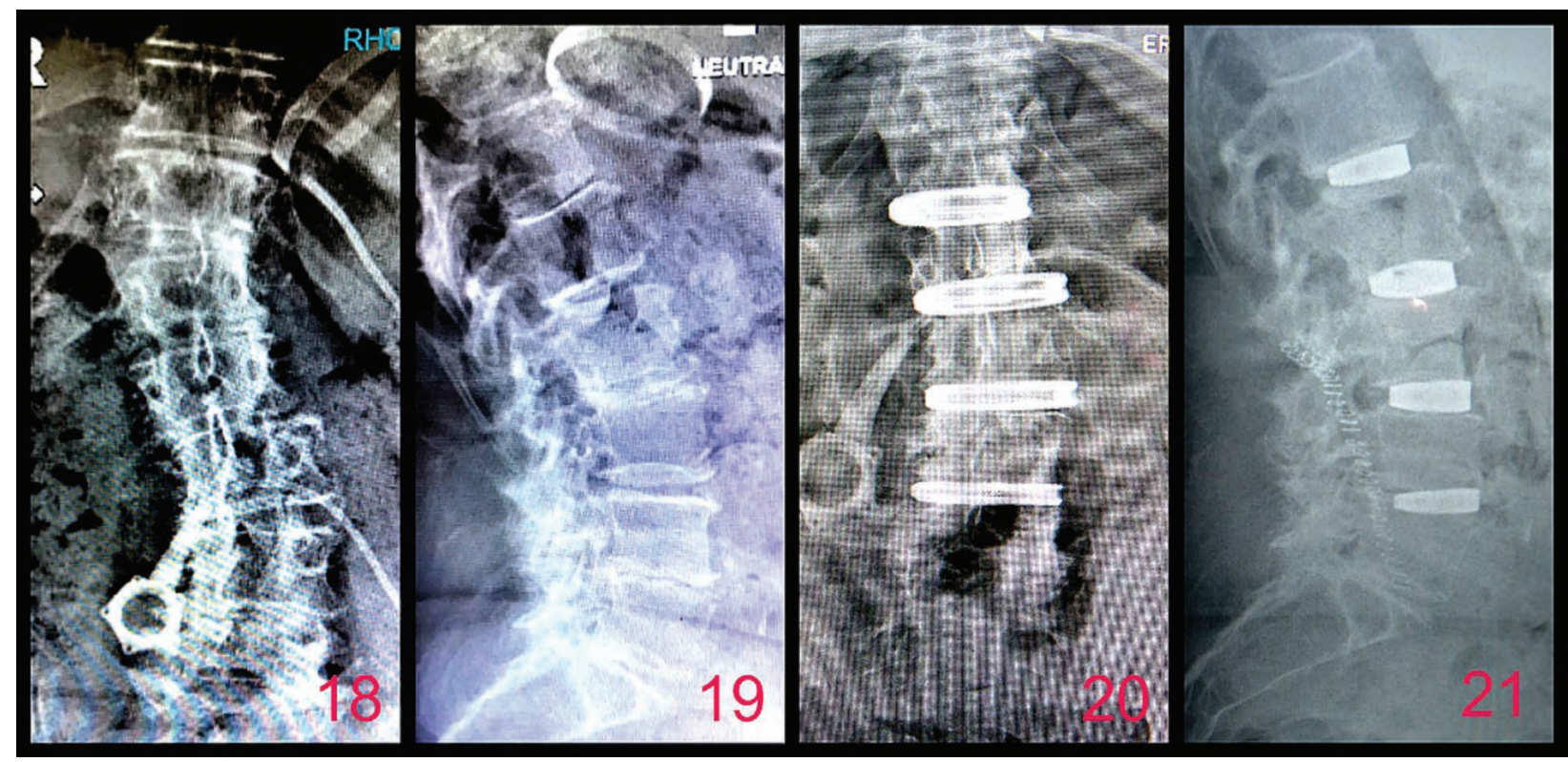




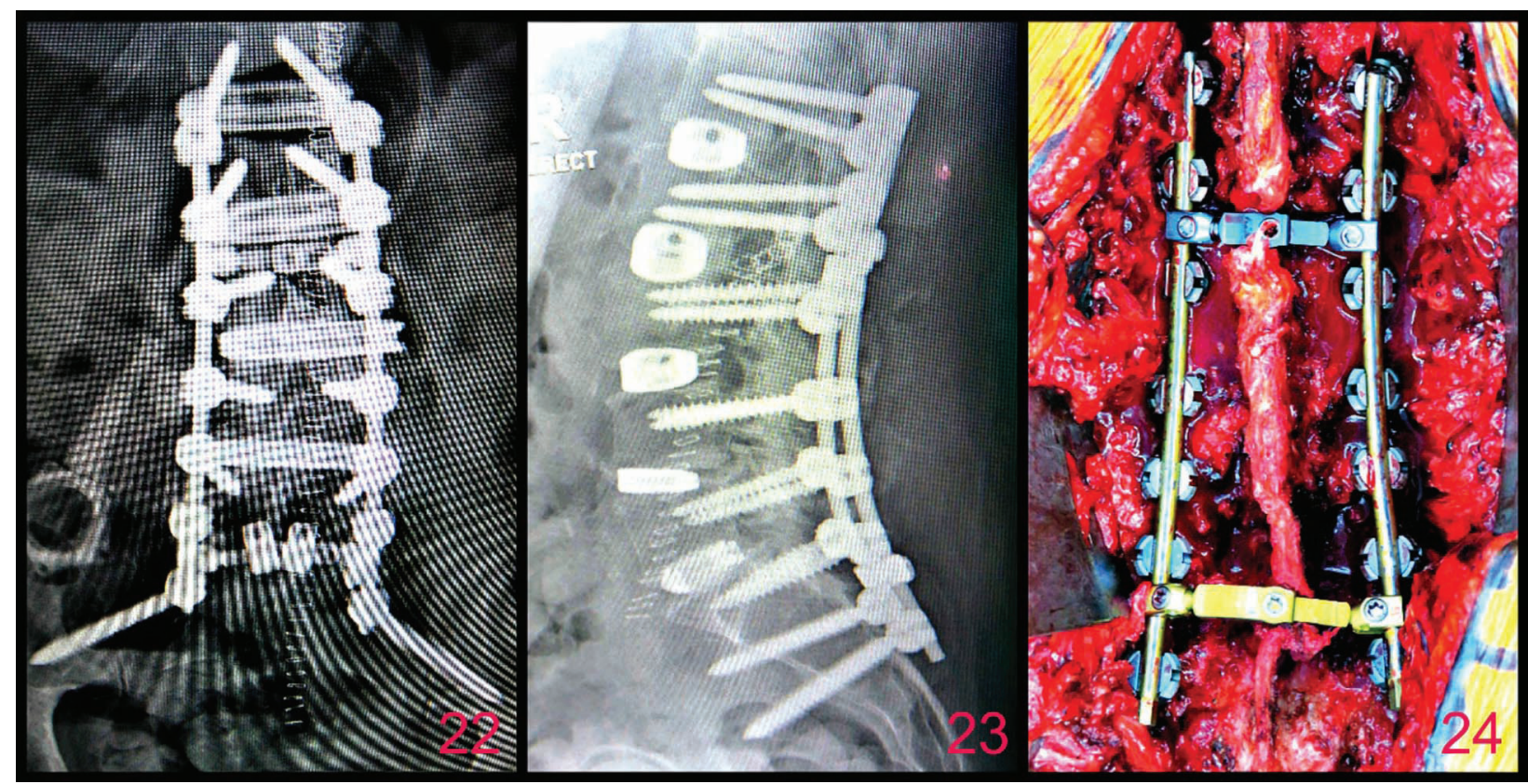

or Cascadia Lat (3D printed titanium)) as the primary procedure for interbody fusion. Almost all patients received supplemental open posterior spinal fusion (PSF) and segmental pedicle screw fixation (K2M Mesa). If LLIF was not possible at L4-5, then PLIF was performed during PSF. If fusion and fixation was required to S1 level, then PLIF L5S1 was performed. PLIF's were performed with PEEK or titanium IBD's (K2M Aleutian or Cascadia). No autograft was harvested from the iliac crest. Bone graft substitute iFactor (Cerapedics) was used in all cases with supplemental local autograft if available. 71 levels were operated in total (2.2 levels per patient). 11 of 32 patients were active smokers (34\%). Average bone mineral index (BMI) was 29.5 (Range 20.038.5). Oswestry disability index (ODI) improved from 36.8 to 23.5. Scoliosis correction went from 4.1 degrees per level to 1.1 degrees per level (a corrective change of 3 degrees per level). All 32 patients fused, therefore the fusion rate was $100 \%$ with no pseudarthroses (personal communication Arvind Dubey). Complications occurred in 4 if 32 patients (12.5\%). Two patients had expulsion of the IBD $(6.3 \%)$ requiring reoperation. One patient had neurologic deficit secondary to fracture of the vertebral body edge requiring reoperation (3.1\%) which subsequently resolved. One patient (3.1\%) had abdominal wall hernia. There were no vascular, bowel, bladder, renal, or ureteral injuries. Conclusions were that LLIF was safe and effective for the indications in this series. Further long term studies are needed to characterize the benefits and risks of LLIF.

A recent case example will serve to illustrate the utility of MIS Ravine LLIF as Stage I surgery, combined with open PSF with Mesa pedicle screws and supplemental PLIF at L5-S1 (Fig. 18-23).

\section{Discussion}

The transpsoas LLIF approach was first described by Luiz Pimenta MD in $2001^{3}$. The technique was developed in conjunction with and commercialized by NuVasive, Inc, San Diego, CA USA. The senior

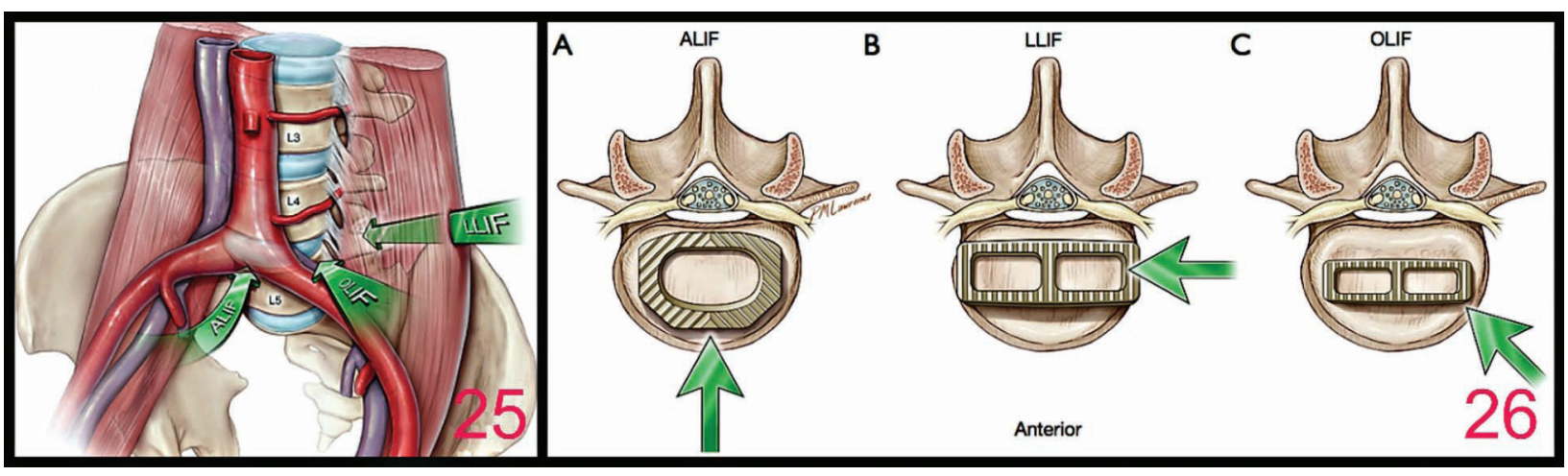


author (CES) was a colleague of Dr. Pimenta's and a consultant for NuVasive at that time. The rationale and logic of the transpsoas approach is confirmed by a plethora of published studies in the literature.

The senior author (CES) began teaching the K2M Ravine LLIF technique in the Asia Pacific region in 2010 as a consultant for K2M. One of the first adopters of the technique in Australia was Dr. Day (MJD). Coincidentally, Dr. Day's clinical nurse practitioner and office manager, J. Luscombe RN (JEL), was a co-developer of the computerized spinal outcomes program with CES, the program known as eFellow (now branded as K2M BACS data collection system). As a result, early outcomes of safety and efficacy of Ravine were confirmed in Dr. Day's practice. Incidentally, Dr. Day, Nurse Luscombe, and their children visited Kathmandu, Nepal as volunteers for Spinal Health International (SHI) in 2013. Dr. Day performed the first spinal operation at Grande International Hospital (GIH) with Binod Bijukachhe MD, GIH Chief of Spine Surgery.

Dr. Dubey of Hobart, Tasmania, Australia began use of the Ravine LLIF system in 2012. An introduction to the technique in the cadaver laboratory was followed by supervised surgical cases in Dr. Dubey's operating theatres, both public and private. Collection of data on all consecutive patients receiving Ravine LLIF procedures was performed from 2012 through 2016 and reported at the Spine Society of Australia annual meeting in Sydney NSW Australia. Nearly all procedures were accompanied by supplemental open posterior fusion and fixation with PLIF performed at L5-S1 and segmental pedicle screw fixation utilizing K2M Mesa complex spine system. Further analysis of this database will investigate comparisons PEEK vs 3D printed titanium IBD's, constructs ending at S1 vs S2AI, traditional anatomic placement of implants vs placement with navigation, and an update of all outcomes for LLIF through 2018.

A recent article of interest in the literature by $\mathrm{Xu}$, Uribe, et al compared the characteristics of the anterior procedures ALIF, LLIF, and OLIF (Fig. 25, $26)^{4}$.

In a systematic fashion, they reviewed the literature, and compared 1. Patient selection, 2. Surgical approach, 3. Outcomes, and 4. Complications for each procedure. The authors concluded that 1 . The anterior procedures of ALIF,
LLIF, and OLIF allow placement of a larger IBD and more bone graft than all posterior procedures, PLIF and TLIF, 2. ALIF is best suited for the L5-S1 level and allows for the largest IBD, however, vascular complications increase at more cephalad levels, 3. LLIF requires neuromonitoring, but is a powerful tool in correction of coronal deformity (scoliosis), however, temporary leg weakness is a recognized risk, 4. OLIF does not require neuromonitoring, but likely has higher risk of subsidence, however, further study is needed.

\section{Conclusions}

Lateral lumbar interbody fusion (LLIF) is an effective and safe procedure. Intraoperative neuromonitoring is recommended for minimally invasive approaches. Positioning of the patient and clear visualization of the operative level(s) by fluoroscopy is critical for successful implantation of the IBD during LLIF. The most useful indication for LLIF is lumbar degenerative deformity (scoliosis and/or loss of lordosis). Most cases should be supplemented by posterior segmental fixation with pedicle screws and rods. It is hoped that this technique can be introduced at spine surgery specialty centers in Nepal, which could be accomplished without neuromonitoring if a miniopen approach is utilized and neurologic anatomy is clearly visualized and protected.

\section{Technical Note - Figure Index}

Fig. 1: Note patient lateral decubitus position on radiolucent operating table. Patient's chest, hips, and legs are taped with proper padding. The table is flexed at the pivot point which is just cephalad of the pelvis. The armboard is positioned to allow entry of the fluoroscope from the anterior aspect of the patient. The surgeon stands posterior to the patient.

Fig. 2: Note the "horizontal" position of the fluoroscopic C-arm provides an anteroposterior (AP) view of the lumbar spine. The disc level to be operated is centered on the fluoroscopic screen to minimize parallax. The fluoroscope is perpendicular to the axis of the spine at the operative level. This allows for a crisp clear view of the vertebral endplates. The pedicles should be located in the upper half of the vertebral body. The spinous process is midway between the pedicles indicating proper rotation. The fluoroscope remains horizontal and any adjustment in rotation is accomplished by tilting the operating table. 
Fig. 3: Note the "vertical" position of the fluoroscopic C-arm provides a lateral (lat) view of the lumbar spine. Again, the disc to be operated is centered on the fluoroscopic screen to minimize parallax and the fluoroscope remains perpendicular to the axis of the spine. This allows for a crisp clear view of the vertebral endplates on lat view as well. The pedicles should be superimposed which denotes proper rotational orientation of the spinal segment to be operated. From this point forward, the fluoroscopic positions should only be horizontal and vertical to allow safe and accurate preparation of the disc and placement of the interbody device (IBD).

Fig. 4: In the vertical position, the skin is marked over the disc space, sterile preparation is made, and the area draped. In the mind's eye of the surgeon, the disc, vertebral, neural, and vascular anatomy is visualized. A minimally invasive surgical (MIS) incision of $2-3 \mathrm{~cm}$ is made. Alternatively, a mini-open incision can be used. The dissection is carried through the fat, muscular, and fascial layers allowing entry to the retroperitoneal space.

Fig. 5: The index finger is inserted into the retroperitoneal space, the space is developed with blunt dissection, and the psoas muscle is palpated.

Fig. 6: A blunt dissector is used to traverse the psoas muscle. Once the tip of the dissector is centered on the disc under fluoroscopic guidance, a large K-wire is tamped into the disc space.

Fig. 7: Intraoperative neuromonitoring (IONM) allows for safe passage of the dissector through the psoas muscle avoiding nerves and nerve roots. Using the K-wire as a guide, a series of dissectors develop a safe passage through the psoas muscle and each dissector utilizes IONM.

Fig. 8: The Ravine frame in the collapsed position is then inserted through the same safe passage by following the K-wire to the disc. The Ravine frame is then expanded to develop the working passage through the psoas muscle, the K-wire removed, and the frame is firmly attached to the vertebral body above and below the disc with 2-4 threaded pins. Ancillary retractors can be placed anteriorly and posteriorly for additional visualization and safety. A supplemental table mount is available for the Ravine retractor frame, but is rarely utilized due to superior stabilization by vertebral fixation pins.

Fig. 9: When fully deployed, fiberoptic light sources are placed in the retractor blades and the disc is visualized and tested with IONM to assure the absence of neural tissue within the operating space.

Fig. 10: Vertical fluoroscopic image is used to confirm proper placement of the Ravine retractor on the lateral surface of the disc prior to disc entry. The surgeon must be confident that the frame is placed accurately to avoid excessive anterior or posterior position which would put vascular and neural structures at risk. The fluoroscope is then moved to the horizontal position to monitor the remainder of the procedure.

Fig. 11: The horizontal fluoroscopic view allows for monitoring the depth of penetration for each instrument used to enter and prepare the disc space and endplates. Once disc preparation is complete, trials are utilized to determine the exact size of the IBD to be inserted.

Fig. 12: The trial will determine length, height, and lordotic angle of the IBD. The width is determined by the vertebral body size and window of entry to the disc space.

Fig. 13: PEEK implants (K2M Aleutian Lateral) are available with a large space available for bone graft material. The tapered nose allows for easy insertion.

Fig. 14: Tantalum markers allow for proper positioning of the IBD under fluoroscopic guidance.

Fig. 15: 3D printed titanium implants (K2M Cascadia Lateral) are also available which allow for bony attachment to and bony ingrowth within the cage itself. Again, a large space is available for bone graft material and the nose is tapered for ease of insertion.

Fig. 16: The titanium implants are easily visualized to determine proper placement within the disc on horizontal fluoroscopic (AP) view.

Fig. 17: The fluoroscope is then changed to the vertical position to confirm proper placement on lateral view. If more IBD's are to be inserted at other levels, then the target disc is centered on the vertical fluoroscopic image and the entire procedure repeated.

Fig. 18: A recent case example reinforces key concepts. AP view demonstrates multilevel degenerative discs with scoliotic deformity in an elderly female with severe progressive low back pain unresponsive to many years of conservative treatment. 
Fig. 19: Lateral view shows disc collapse at each level and modest lordosis.

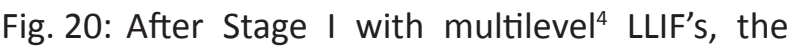
scoliotic deformity is much improved. Scoliotic deformity correction is the principal advantage of the lateral approach. In addition, large IBD's are placed at each level with a large volume of bone graft material.

Fig. 21: Lateral view after Stage I demonstrates increased disc height and improved neuroforaminal dimensions at each level with maintained lordosis.

Fig. 22: After Stage II, segmental pedicle screw fixation and further correction of scoliosis is achieved. The construct is well anchored caudally with convergent S1 pedicle screws and divergent $\mathrm{S} 2 \mathrm{Al}$ pelvic fixation. A crosslink between S1 and S2Al adds considerably to the pullout strength of this cluster of screws. The second crosslink in a cephalad location contributes to rotational stability of the entire construct.

Fig. 23: Lateral view shows proper screw position at each level and added lordosis.

Fig. 24: Intraoperative photograph illustrates the posterior implant construct. Meticulous decortication of all bony surfaces and voluminous bone graft material completes the procedure. Local autograft can be utilized with bone graft substitute, but harvesting of autograft from the iliac crest is rarely performed. At least two crosslinks are highly recommended in complex lumbar scoliotic deformity surgeries, and three or more as the construct extends to thoracic vertebral levels.

Fig. 25: Illustration from Uribe's article demonstrating the three basic anterior approaches to the lumbar spine.

Fig. 26: Illustration from Uribe's article demonstrating the relative IBD size and bone graft space for each of the anterior lumbar approaches. As techniques have improved with OLIF, it is now possible to insert larger IBD's by this approach.

\section{Acknowledgements}

Thank you to Anna De Winter of LifeHealthcare Pty Ltd, Hobart, Tasmania, Australia for assistance with collection of patient lists and implant data. Thank you to Olivia Day, University of Technology Sydney, School of Nursing, Australia for photographs of $\mathrm{GIH}$ operating theater. No financial support was received for production of this manuscript.

Conflict of interest: None of the authors has a conflict of interest related to the products discussed in this manuscript. C. Sutterlin, MD receives royalties from $\mathrm{K} 2 \mathrm{M}$ for unrelated implants; is an educational consultant for LifeHealthcare in Australia and New Zealand; and has stock ownership in eFellow (aka K2M BACS Data Management System). J. Luscombe, $\mathrm{RN}$ is a consultant for LifeHealthcare (Australia and New Zealand distributor for K2M) and shares her expertise related to eFellow (aka K2M BACS Data Management System) for education and training of spine surgical practices in Australia and New Zealand.

\section{References}

1. Briggs $H$, Milligan, PR. Chip fusion of the low back following exploration of the spinal canal. JBJS 1944;26:125-30.

2. Asad S, Dubey A, Dubey A, Sutterlin C. Clinical outcomes after minimally invasive trans-psoas lateral interbody fusion for the treatment of adult degenerative scoliosis: 4 years' multicenter study. (Publication pending)

3. Pimenta L. Lateral endoscopic transpsoas retroperitoneal approach for lumbar spine surgery. VIII Brazilian Spine Society Meeting, Belo horizonte, Minas Gerais, Brazil, May, 2001.

4. Xu DS, Walker CT, Godzik J, Turner J, Smith W, Uribe J. Minimally invasive anterior, lateral, and oblique interbody fusion: A literature review. Ann Transl Med. 2018;6(6):104. 